\title{
Risikoselektion ist in der GKV unzulässig
}

Gesetzliche Krankenkassen haben nicht das Recht, ihre Versicherten systematisch aus bestimmten Personenkreisen anzuwerben. Diese Praxis verstößt gegen das Diskriminierungsverbot und das Solidarprinzip der Gesetzlichen Krankenversicherung (GKV). Dies betonte die Bundesregierung in ihrer kürzlich veröffentlichten Antwort auf eine parlamentarische Anfrage der Linksfraktion im Bundestag.

$\mathrm{Zu}$ den nicht zulässigen Zielgruppenvereinbarungen gehören demnach Absprachen, die Aufwandsentschädigungen oder Prämien für die Werbung bestimmter Mitglieder umfassen. In der Antwort heißt es, das Verbot gelte unabhängig davon, ob die sogenannte Risikoselektion auf Grundlage von Einkommensgruppen oder der Zugehörigkeit zu einem bestimmten Personenkreis erfolge. Unterschieden werden müsse jedoch zwischen Zielgruppenvereinbarungen und allgemeinen Werbemaßnah- men mit Angeboten für einen bestimmten Personenkreis. Werbemaßnahmen seien erlaubt, wenn sie nicht potenzielle Mitglieder ausschlössen oder das Recht zur Kassenwahl einschränkten, heißt es im Schreiben der Bundesregierung. Das Bundesversicherungsamt habe Zielgruppenvereinbarungen in der Vergangenheit konsequent aufsichtsrechtlich aufgegriffen und werde dies auch künftig tun. Bonusprogramme der Kassen müssten sich an alle Mitglieder richten und diskriminierungsfrei ausgestaltet sein. Die Linksfraktion hatte sich in ihrer Anfrage auf einen im Februar in der Ärzte Zeitung veröffentlichten Artikel berufen. Dort hieß es, dass der Zeitung interne Dokumente vorlägen, „wonach der Anreiz, insbesondere gesunde Gutverdiener anzusprechen, in den Vertriebsabteilungen der Kassen nicht nur in den Hinterköpfen präsent ist“.

\section{Unlauterer Wettbewerb}

\section{Zentrale moniert Verstöße von Krankenkassen}

Die Zentrale zur Bekämpfung unlautereren Wettbewerbs hat im ersten Quartal des Jahres bei 14 von 20 bearbeiteten Fällen Verstöße der Krankenkassen gegen den Wettbewerb festgestellt. Darunter fallen vor allem irreführende Werbung und aggressive geschäftliche Handlungen. Dies gab die Wettbewerbszentrale im April in einer Zwischenbilanz bekannt.

In zwei Fällen erhob die Kontrollinstitution sogar eine Unterlassungsklage. Außerdem beantragte sie einmal eine einstweilige Verfügung und erteilte in vier Fällen Hinweise. Bei irreführender Werbung ging es vor allem um Zusatzleistungen, mit denen sich die Kassen in der Öffentlichkeit schmückten. Als Beispiel nannte die Wettbewerbszentrale die Werbepraktiken der AOK im Zusammenhang mit der professionellen Zahnreinigung (PZR). Nach Angaben der Wettbewerbswächter hatte die Kasse mit dem Versprechen „Ab jetzt noch mit mehr Leistungen“ geworben. Dabei habe die AOK allerdings nicht erwähnt, dass sie anteilig 40 Euro erstattet und damit nicht die kompletten Kosten übernimmt. In diesem Fall verpflichtete sich die Kasse gegenüber der Wettbewerbszentrale zur Unterlassung.

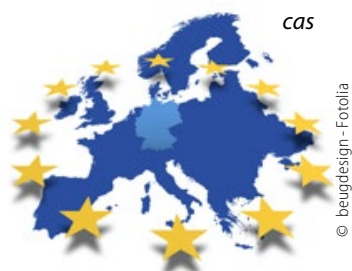

Die Europäische Union (EU) und 26 Mitgliedstaaten haben ein durch das Umweltprogramm der Vereinten Nationen (UNEP) ausgehandeltes neues internationales Übereinkommen über Quecksilber unterzeichnet. Es betrifft den gesamten Lebenszyklus von Quecksilber vom primären Quecksilberbergbau bis zur Quecksilberabfallentsorgung und zielt darauf $a b$, die menschliche Gesundheit und die Umwelt vor Emissionen von Quecksilber und Quecksilberverbindungen in die Luft, dem Wasser und dem Boden zu schützen.

Zur Umsetzung des Abkommens hat die EU-Kommission einen Vorschlag für eine Verordnung des Parlaments und des Rates über Quecksilber und zur Aufhebung einer bestehenden Verordnung vorgelegt. In diesem Vorschlag führt die EU-Kommission unter anderem aus: „Die Folgenabschätzung führt anhand der verfügbaren wissenschaftlichen Informationen $\mathrm{zu}$ dem Schluss, dass ein Verbot der Verwendung von Dentalamalgam nicht verhältnismäßig wäre, da die von Dentalamalgam ausgehenden Gesundheitsrisiken nicht eindeutig nachgewiesen sind und ein Verbot hohe Kosten mit sich bringen würde." Die Folgenabschätzung habe gezeigt, dass zwei Maßnahmen aus der vorgeschlagenen Maßnahmenliste (Beschränkung der Verwendung von Dentalamalgam auf dessen verkapselte Form und Förderung des Einsatzes der besten Umweltschutzpraktiken in zahnmedizinischen Einrichtungen), Umwelt- und Gesundheitsvorteile zu geringen Kosten erbringen würden.Diese Maßnahmen würden die Exposition von Zahnärzten und Patienten gegenüber Quecksilberemissionen mindern und eine erhebliche Verringerung der Freisetzungen von Quecksilber in die Kanalisation und die Umwelt über kommunale Kläranlagen gewährleisten. Ferner werde damit gerechnet, dass in Unternehmen, die Amalgamabscheider herstellen, installieren und warten oder auf die Sammlung und Behandlung von quecksilberhaltigen Abfällen spezialisiert sind, neue Arbeitsplätze entstehen werden. Aktuelle Rechtssprechung dazu auf Seite 52 red/brx 